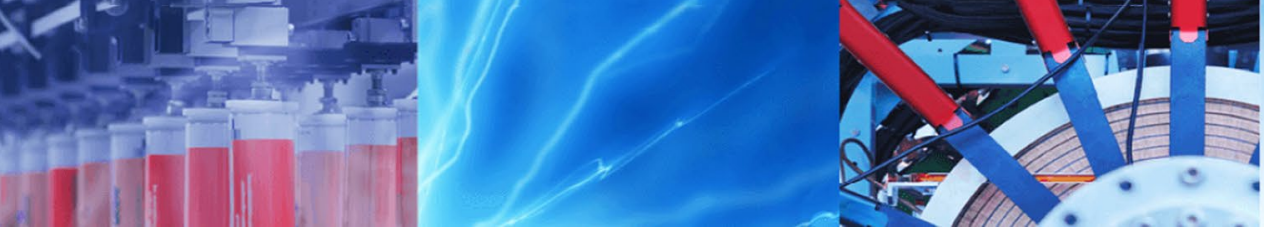

Research Article

\title{
Elastic three-dimensional graphene sponge fabricated by the liquid crystals of controlled large graphene oxide sheets
}

\author{
Q. Wei ${ }^{1} \cdot$ A. Wufuer $^{1} \cdot$ M. Wang ${ }^{1} \cdot$ Y. Wang $^{1}$ (D) $\cdot$ L. Dai $^{1}$
}

(c) Springer Nature Switzerland AG 2018

\begin{abstract}
Three-dimensional graphene (3DG) sponge has attracted increasing attention because it combines the unique properties of cellular materials and the excellent performance of graphene. Preparation of 3DG sponge depends mainly on the self-assembly of graphene oxide sheets. In the case of using uniform large graphene oxide and ultralarge graphene oxide sheets, the nematic liquid crystals (LCs) phases are formed at low concentration. After chemical reduction, the LCs of $\mathrm{GO}$ solution are converted to 3DG sponges with a high degree of orientation, offering a new methodology to regulate the controlled large GO sheets. The orientation of GO solution can be inherited by 3DG sponge, making the sponge to have a large-scale ordered network structure. The 3D elastic graphene sponges have low density and good elasticity, promising for the applications in strain sensing, shock damping, and energy cushioning. Our work explores a novel strategy for organizing the ordered alignment of controlled large GO sheets and exploring the relationship between the microstructures and mechanical properties of 3DG sponge.
\end{abstract}

Keywords Elastic · Three-dimensional · Liquid crystals · Graphene sponge · Self-assembly

\section{Introduction}

The Graphene, a single atomic plane of graphite, can be well used as the building block of graphene-based macroscopic materials [1, 2]. Recently, the graphenebased macroscopic materials such as one dimensional graphene (1DG) fiber, two dimensional graphene (2DG) paper [3], and three-dimensional graphene (3DG) sponge have attracted significant attention as a means of further expanding the significance of graphene $[4,5]$. Because GO sheets are a precursor for the cost-effective and mass production of graphene-based materials, the lateral dimensions of GO sheets play an important role in determining the structures and properties of graphene-based macroscopic materials [6].
The large GO sheets are ideally suited for the preparation of ultrastrong 1DG fibers [7], highly aligned 2DG papers $[8,9]$. In these cases, their better alignments are the main factors to improve the mechanical performance [10]. The liquid crystals (LCs) of GO sheets with regular ordering provides the most viable fluid assembly approach. It is an important precursor for fabrication of high performance aligned graphene-based macroscopic materials. Therefore, the LCs suspensions of high aspect ratio GO are of strong practical interest. From a fundamental point of view, the LCs of GO sheets could be the closest experimental realization of theoretical models based on infinitely thin and high aspect ratio rigid platelets $[6,11,12]$. The 1 DG fiber and 2DG paper prepared with the LCs of controlled large $\mathrm{GO}$ sheet $\mathrm{s}$ have excellent mechanical properties $[7,8]$.

Electronic supplementary material The online version of this article (https://doi.org/10.1007/s42452-018-0062-3) contains
supplementary material, which is available to authorized users.

Y. Wang, ecnu_yywang@163.com | 1 College of chemistry and Molecular Engineering, East China Normal University, 500 Dongchuan Road, Shanghai 200241, China.

SN Applied Sciences (2019) 1:109 | https://doi.org/10.1007/s42452-018-0062-3

Received: 13 September 2018 / Accepted: 15 November 2018 / Published online: 18 December 2018 
Therefore, the 3DG sponge constructed with these systems have huge potential in further research.

Due to its high flexibility, size heterogeneity, random distribution of functional groups and disorderly stacking of the GO board, it is a great challenge to accurately arrange the controlled large $\mathrm{GO}$ board and control the restore process in 3D architecture. [13]. It is known that fluid phase assembly or the formation of lyotropic LCs is one of the most viable approaches to obtain large-scale, ordered microstructures from nanoscale building blocks [14]. The forming of $L C s$ of $G O$ sheets depends on the sizes distribution, concentration of GO solution and the viscosities of solvents [6]. The liquid crystalline phase is produced easily with concentrated $\mathrm{GO}$ constructed using large size sheets $[15,16]$. Nevertheless, the ordered 3DG sponge produced with LCs of concentrated large GO sheets will lose its advantage of low weight densities. An excellent work by Shi et al. addresses this issue to some extent. They successfully regulate the arrangement of $G O$ sheets via increasing its $\mathrm{pH}$ value with potassium hydroxide $(\mathrm{KOH})$ [13]. The 3DG sponge constructed using the LCs of GO sheets [13] and large GO sheets [17-19] are reported recently. However, the ordered structure of 3DG sponge fabricated by controlled large GO sheets has been rarely discussed.

This paper reports a chemical reduction method to prepare elastic and low-density 3DG sponge fabricated by highly oriented LCs of large graphene oxide (LGO) and ultralarge graphene oxide (ULGO) sheets [11]. The LGO and ULGO sheets with extremely high aspect ratios as building blocks reduce defective edges, and achieve highly ordered alignment of rGO sheets in 3DG sponge. This work provides a new method for the preparation of 3DG materials constructed using LCs of controlled large GO sheets and the mechanical properties of 3DG sponge are expected to be improved. The method may shed new light on the relationship between the microstructures and mechanical properties of 3D graphene assemblies.

\section{Materials and method}

\subsection{Materials and reagents}

Natural Graphite (AR), KOH(AR) was purchased from Aladdin Company. $\mathrm{HCl}$, L-ascorbic acid are supplied from Sinopharm Chemical Reagent Co. All chemical reagents are used without further purification. Deionized water is used throughout the experiments.

\subsection{Preparation of LCs of controlled large GO sheets}

The SGO, LGO and ULGO solution were prepared by different natural graphite flakes with 32, 325 and 1200 mesh.
The process included three steps: oxidization, size fractionation, and exfoliation. In the first step, the three kinds of GO sheets were oxidized by a modified Hummer method. In the second step, the mixture was repeatedly washed with $\mathrm{HCl}$ solution $(1: 10)$ and water, after the process of oxidation. The GO dispersion was centrifuged at $1100 \mathrm{rpm}$ for 3 min to separate into two portions: large and small lateral dimensions. Larger GO (32 and 325 mesh) left the larger part, while the smaller portion was left by 1200 mesh. The low speed centrifugation process was repeated three times for narrowing the size distribution. Then, it was purified by dialysis for 1 week. In the third step, graphite oxide was exfoliated by the freeze-thaw method [13]. The LCs of controlled large GO sheets were formed with LGO and ULGO solution $(5 \mathrm{~g} 0.3 \%)$ with the adding of $\mathrm{KOH}(0.014 \mathrm{M})$ respectively.

\subsection{Preparation of 3DG sponge and three-dimensional elastic graphene 3DEG sponge}

These two kinds of LCs of large GO solution constructed with LGO and ULGO sheets were put in the glass bottle through freeze-drying process. The GO sponges were obtained. The 3DG sponges were prepared by mild chemical reduction. The ascorbic acid was added in the LCs of GO solution ( $0.3 \%$ ) constructed using LGO and ULGO sheets respectively. The ratio of ascorbic acid to $G O$ was double. Then, the bottles were maintained at $90^{\circ} \mathrm{C}$ for $1 \mathrm{~h}$. The 3DG sponges were obtained. In order to prepare the elastic materials, the network of 3DG sponge should be strengthened by one more step. The LCs of GO solution were freezing in dry ice before chemical reduction. Then, these samples were maintained at $90{ }^{\circ} \mathrm{C}$ for $1 \mathrm{~h}$. The 3D EG sponges prepared by LGO and ULGO solution were obtained.

\subsection{Characterization}

X-ray diffraction (XRD) patterns of the Pt-Cu@3DG were performed on a Bruker D8 diffractometer with $\mathrm{Cu} \mathrm{Ka}$ radiation with a scan speed $10^{\circ} \mathrm{min}^{-1}$. Scanning electron microscopy (SEM) was performed on a Philips XL 30 microscope operating at $30 \mathrm{kV}$.

\section{Results and discussion}

\subsection{The formation of LCs of GO solution at low concentration}

The LCs behavior of aqueous GO dispersion depends on the sizes distribution and concentration of $\mathrm{GO}$ sheets 
$[18,20]$. The effect of these two aspects were discussed below. There are three kinds of GO sheets. The GO sheets were mostly smaller than $20 \mu \mathrm{m}$ in small graphene oxide (SGO), 20-43 $\mu \mathrm{m}$ in LGO, and larger than $60 \mu \mathrm{m}$ in ULGO (Fig. S1). A low concentration of GO dispersion (0.3\%) was immobilized for a sufficiently long time (usually more than 3 weeks) and macroscopically phase-separated into two phases was formed [21]. The SGO solution still kept uniform dispersions because of the smaller size distribution of GO sheets, belong to isotropic phase (Fig. 1a). To the naked eye, the as-prepared ULGO dispersions exhibited an inhomogeneous dark chocolate-milk like wavy appearance which might be mistaken for precipitation. In fact, it was an indication of nematic LC phase due to its high viscidity and ultralarge sheet size, as shown in the inset (Fig. 1c) $[22,23]$. The LGO solution was separated into two phases, as shown in the inset (Fig. 1b). The low-density top phase was isotropic and the high-density bottom phase was demonstrated nematic [18, 21]. We provided respective models for their isotropic and nematic phases as shown in
Fig. 1. The GO sheets showed a chaotic distribution in $\mathrm{SGO}$ solution and ordered alignments in the ULGO solution. The model of biphasic phase transition of LGO solution was proven in Fig. 1b. The LCs solution was formed when the concentration of LGO dispersion was higher than $7 \mathrm{mg} \mathrm{g}^{-1}$ (Fig. 1d) [23].

In order to decrease the concentration of LCs solution, $\mathrm{KOH}$ was induced. From GO sheets to 3DG sponge, the principle schematic of forming process was showed in Fig. 2. The rearrangement of $\mathrm{GO}$ sheets was guided upon adding the base. During the reduction of GO solution, the sheets was aggregated in a wavelike form. The effect of base was studied by characterizing the structure of $\mathrm{GO}$ sheets and 3DG sponge. The GO sheets were partially deoxygenation in an alkaline solution to restore their conjugated structures. The reduction was confirmed by the darkening of the solution upon adding this base (inset of Fig. 2b) and the changes of peak of 3DG sponge with different content of base. The XRD pattern of 3DG sponge (Fig. 2b) exhibited a sharp (002) reflection peak at $2 \theta=23^{\circ}$.
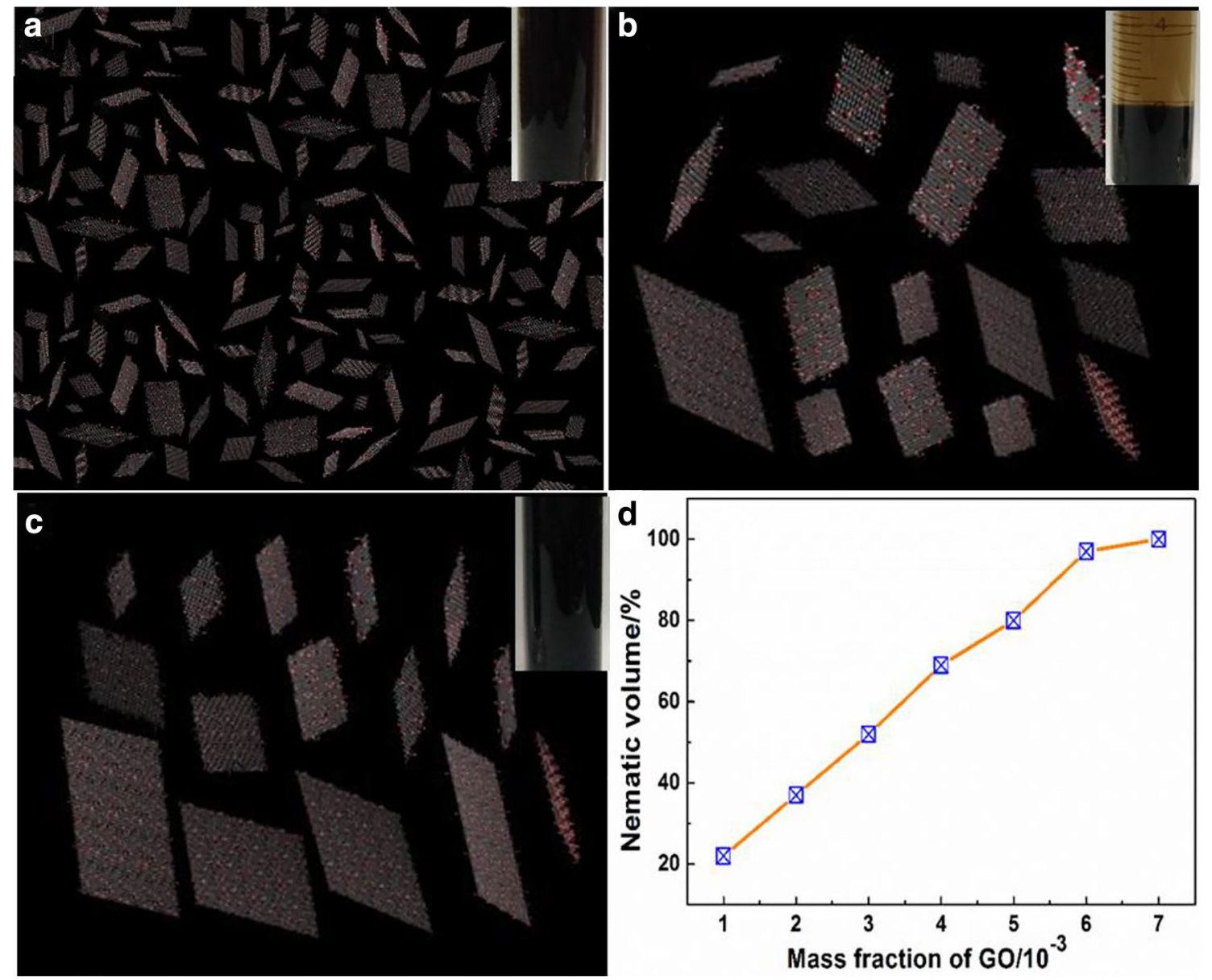

Fig. 1 Schematic models and photographs (in the inset) of isotropic, biphasic and nematic LC phase of SGO (a), LGO (b), and ULGO (c) solution respectively after phase separation. $\mathbf{d}$ The relative

volume of the nematic phase after phase separation as a function of LGO mass fraction, revealing three phase states include isotropic, biphasic and nematic phases along with the mass fraction 

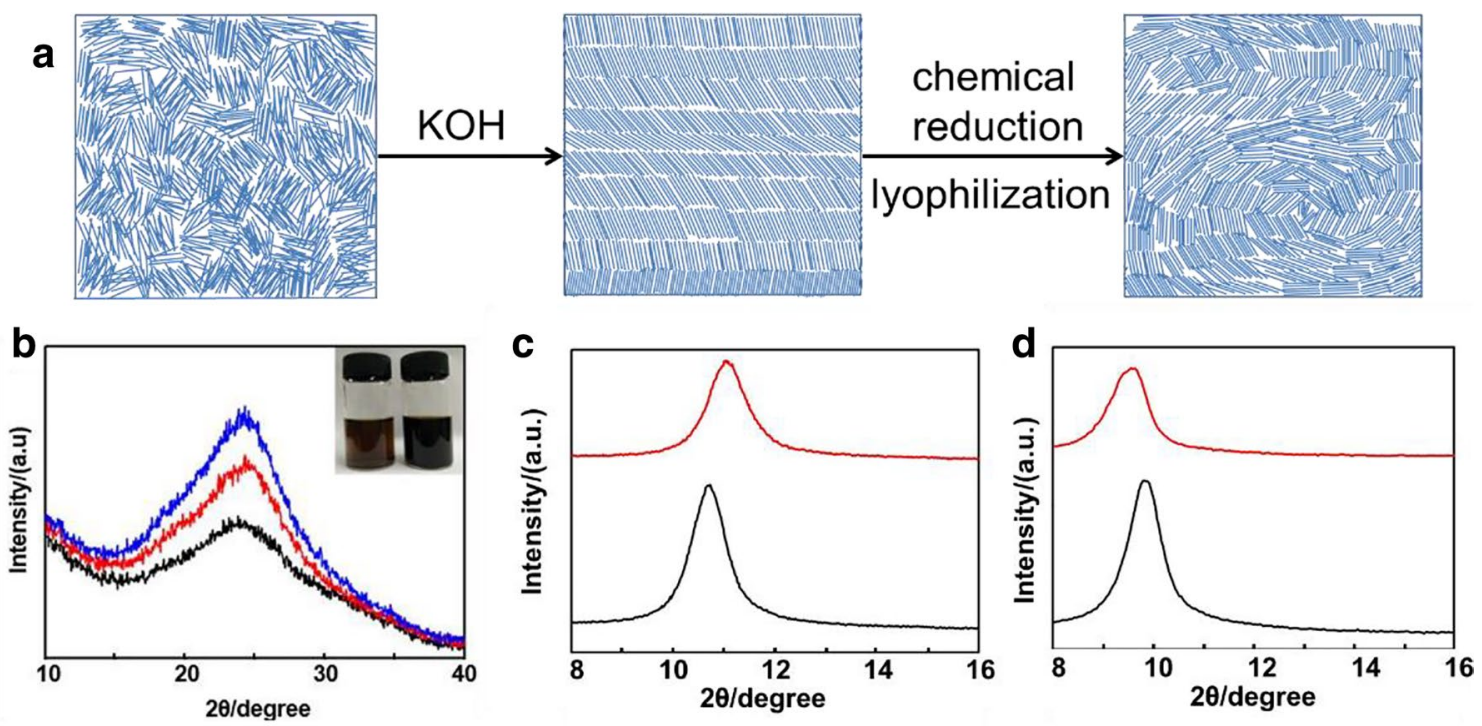

Fig. 2 a Schematic illustration of the fabrication of 3DG. b XRD tographs (inset) of $\mathrm{GO}$ and $\mathrm{GO}+\mathrm{KOH}$ solution. XRD patterns of $\mathrm{GO}$ patterns of 3DG sponge prepared with LGO (black), LGO $+\mathrm{KOH}$ sponge (c) and LCs GO sponge (d) (LGO (red) and ultra-LGO (black)) $(0.014 \mathrm{M})$ (red) and LGO $+\mathrm{KOH}(0.018 \mathrm{M})$ (blue) solution; the pho-
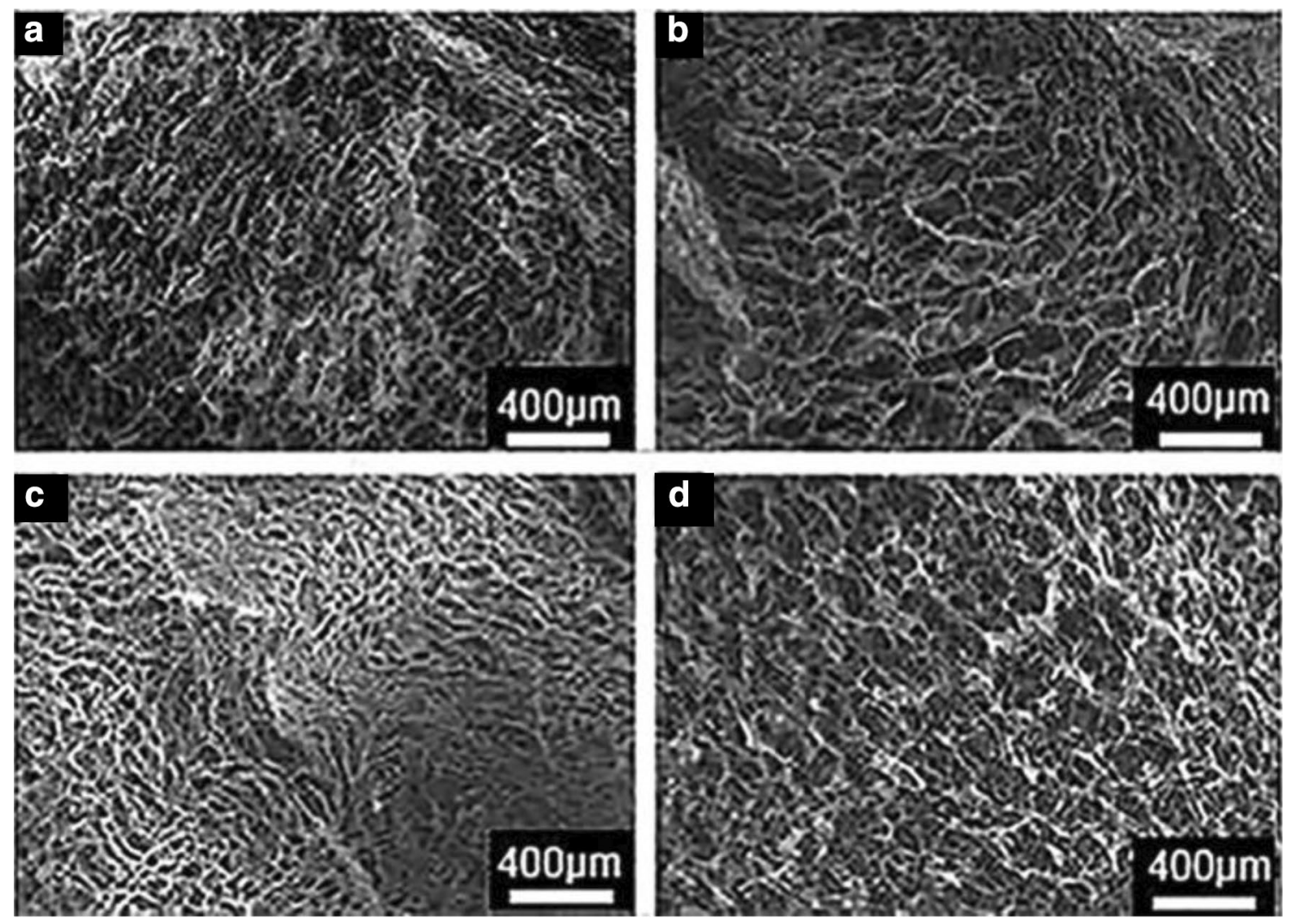

Fig. 3 SEM images of GO sponge ( $\left.8 \mathrm{mg} \mathrm{g}^{-1} \mathrm{GO}\right)(\mathbf{a}, \mathbf{b})$ and LC GO sponge ( $\left.3 \mathrm{mg} \mathrm{g}^{-1} \mathrm{GO}\right)(\mathbf{c}$, d) prepared with ultra-LGO (a, c) and LGO (b, d) sheets 

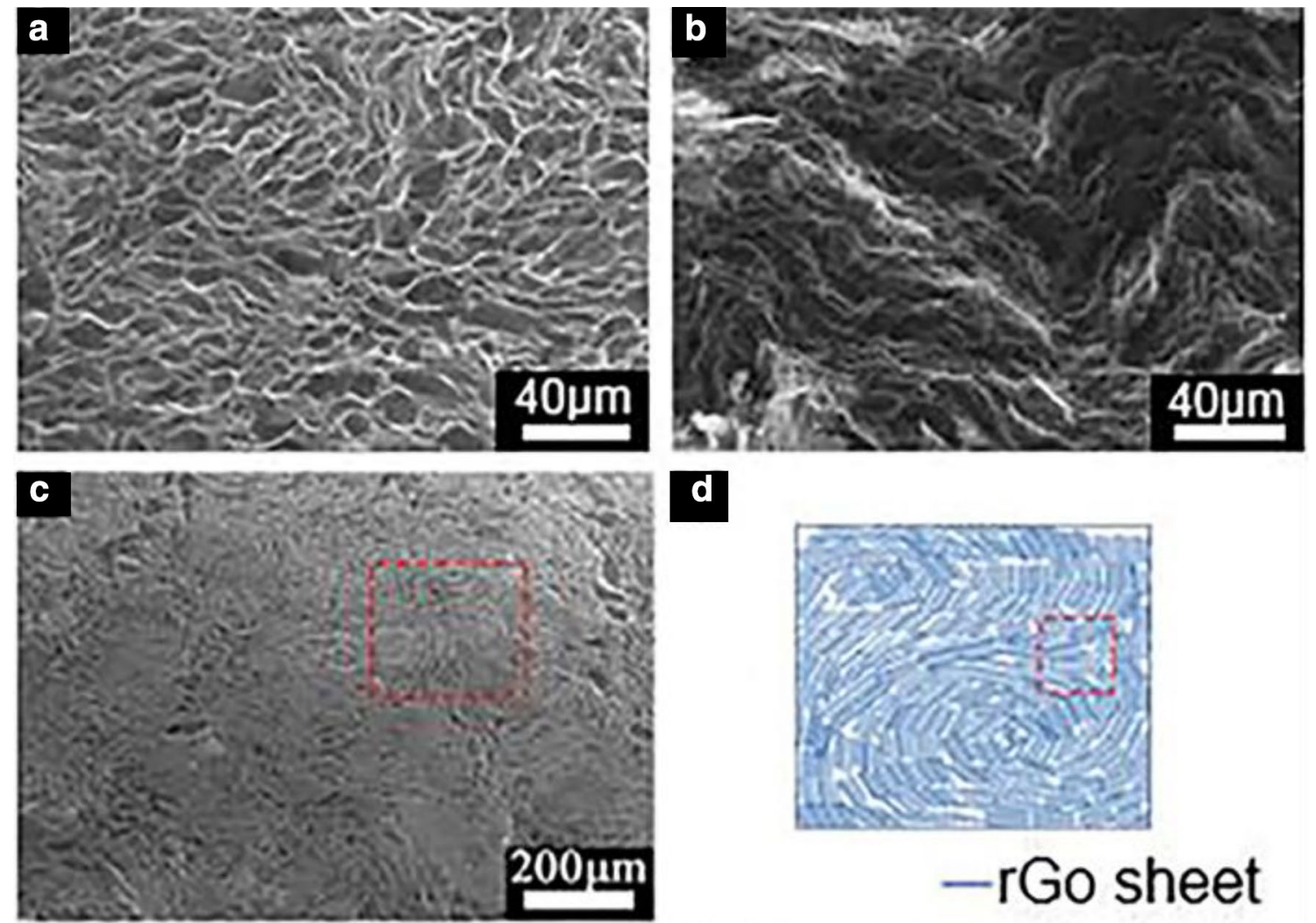

d
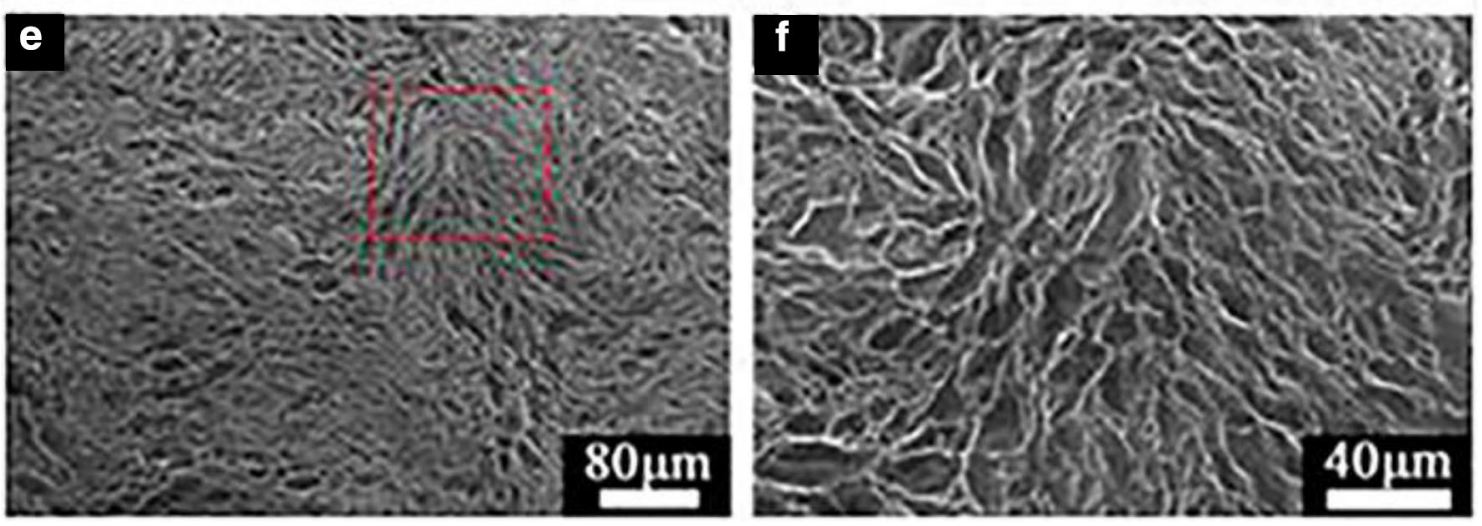

Fig. 4 SEM images of 3DG sponge constructed with ULGO (a) and LGO (b) sheets. SEM images of 3DG sponge constructed with ULGO in a wavelike form with different magnifications (c, e, f). Schematic

The more content of $\mathrm{KOH}$ added, the stronger (002) peak was. The oxygen-containing groups existed on the GO sheets were reduced heavily with more base.

The GO sheets with more oxygenated groups were more flexible, which formed more wrinkled morphology and influenced the formation of LCs phase [24]. The treatment with $\mathrm{KOH}$ extended the rigid domains of $\mathrm{GO}$ sheets, enabling them to self-assemble into a highly ordered structure. However, the order degree of LCs solution was insufficient and the large-scale ordered arrangement could not be obtained in GO sponge with inadequate illustration of the arrangement of graphene sheets in 3DG sponge. The images in panels $\mathbf{c}$ and e correspond to the area in scheme (d)

amount of base (Fig. S2a). The fewer oxygenated groups with excess base led to stacking behavior of sheets and influenced the ordered arrangement in 3DG sponge (Fig. S2b).

\subsection{The ordered structure of $\mathrm{GO}$ sponge}

The aggregation extent of GO sponge could be characterized by XRD analysis with the value of $d$-spacing. The lamellar spacing depended on the content of oxygencontaining groups or spaces existed in the sponge. The 

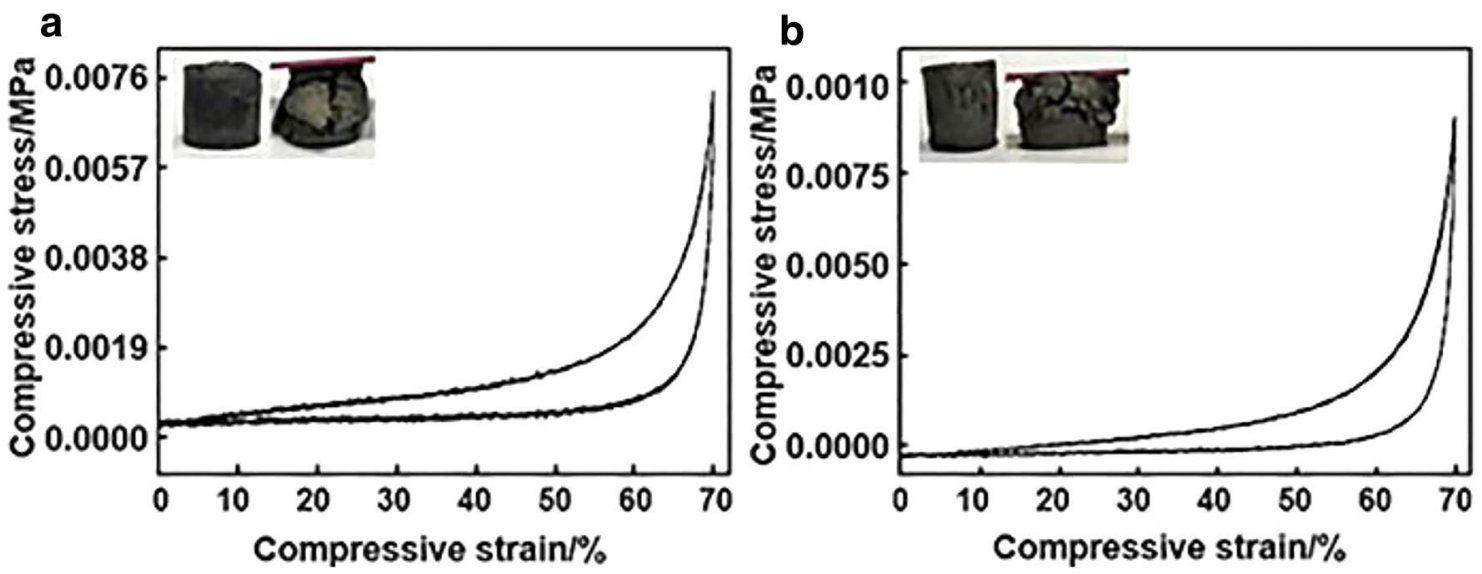

Fig. 5 Compressive stress-strain curves of 3 cycles of loading and unloading of 3D EG sponge prepared with crude ULGO (a) and LGO (b)

two kinds of $\mathrm{GO}$ sponges without the adding of $\mathrm{KOH}$ were prepared with LGO and ULGO solution and their d-spacing were calculated to be $8.01 \AA$ and $8.23 \AA$, correspondingly (Fig. 2c). The d-spacing between the adjacent GO sheets showed a rising trend with growing sheet size. In this case, the main reason was determined by the spaces existed in $\mathrm{GO}$ sponge due to the unordered stacking of $\mathrm{GO}$ sheets. The $\mathrm{GO}$ sponges with the adding of $\mathrm{KOH}$ were prepared with LGO and ULGO solution and their d-spacing were $9.24 \AA$ and $9.01 \AA$, respectively (Fig. 2d). The variation tendency indicated that the $d$-spacing was decided by the number of oxygen-containing functional groups in GO sheets. This result was explained by the ordered and large-scale network structure in GO sponge characterized by SEM (Fig. 3c, d).

There was another way to form GO sponge with the LCs of concentrated $\mathrm{GO}$ solution $\left(7 \mathrm{mg} \mathrm{g}^{-1}\right)$ after lyophilization (Fig. 3a, b). By contrast, the GO sponge prepared with LCs of $\mathrm{GO}$ solution ( $3 \mathrm{mg} \mathrm{g}^{-1}$ ) had larger scale network due to the ordered arrangement of LCs solution upon adding the base (Fig. 3c, d). The enhanced electrostatic repulsion between $\mathrm{GO}$ sheets by excess $\mathrm{KOH}$ prevented sheets from precipitation and stacking each other, enabling them to form an ordered structure in LCs. The enhanced electrostatic repulsion also increased the fluidity of a GO solution. Furthermore, the increased fluidity facilitated tuning the direction vector of $L C s$, realizing rational regulation of the arrangement of GO sheets [16].

\subsection{The ordered structure of 3DG sponge}

The LCs of GO solution with ordered arrangement was reduced by ascorbic acid. After chemical reduction [25], the $\mathrm{GO}$ solution was converted to 3DG sponge with $\mathrm{rGO}$ sheets orderly organization as shown in Fig. $4 a$, b. The forming process of 3DG sponge was explained in Fig. $2 a$.
The reduced part of GO sheet was hydrophobic and another part with oxygen-containing groups was hydrophilic. These sheets turned to the different directions, because the distribution of oxygen-containing groups was asymmetrical dispersed in the GO sheet. The rGO walls were bridged with each other. When the mixture was reducing at $90^{\circ} \mathrm{C}$, the $\mathrm{LCs}$ of $\mathrm{GO}$ solution with ordered arrangement were reduced and showed slightly deformation (Fig. 4d). The resulting materials possessed wavelike microstructures with orderly organized rGO sheets (Fig. 4e, f). The forming of 3DG sponge had a mild reaction condition with the adding of reducing agent, heating to $90^{\circ} \mathrm{C}$ at constant pressure. However, it was difficult to shape into 3D structure, if the reducing agent was lesser. When the reducing agent was more, the reducing speed was too fast to keep the ordered arrangement of sheets (Fig. S3). The orderly arrangement of LCs should be kept with the adding of reducing agent. The ranges of the content of ascorbic acid were tested to be 2-3 times of the mass of $\mathrm{GO}$, which were suitable for the forming of oriented morphology.

\subsection{The elastic property of 3D elastic graphene (EG) sponge}

The preparation of 3D EG sponge required one more step-freeze drying. The LCs of GO solution was freezing in dry ice before chemical reduction. The network was strengthened by this treatment and further freeze was found to have little effect on the structure of network [26]. The freezing LCs of GO solution was reduced by chemical reduction, meanwhile the sheets can keep the ordered arrangement. The microstructures of 3D EG sponge was shown in Fig. 6a, b. The pore structure showed a linear arrangement and the pore size was larger comparing with 3DG sponge. These structural characteristics made the 3D 
EG sponge have a good elastic property. The 3D EG sponge prepared by crude LGO and ULGO solution showed a poor elastic properties comparing with the LCs of GO solution. The materials without the LCs of GO sheets were tested by cycling compression and broken for 2-3 cycles (Fig. 5). It indicated that the ordered arrangement of $\mathrm{rGO}$ sheets play an important role in determining the mechanical property of 3DG sponge.

The elasticity of 3D EG sponges prepared by the LCs of $\mathrm{GO}$ solution were tested by cycling compression and relaxing at a strain rate of $100 \% \mathrm{~min}^{-1}$ (Fig. 6c, d). Compression tests revealed that the 3D EG sponge exhibited excellent resilience when released from compression. Three regimes of deformation could be observed in the loading stress-strain curve: nearly linear elastic regime, corresponding to bending of the cell walls; relatively flat stress plateau, corresponding to elastic buckling of cell walls; and abrupt stress increasing regime, corresponding to densification of cells (Fig. 6c, d) [26, 27]. For the first compression cycle, the nearly linear elastic region of the sponge extended up to $20 \%$ strain. The plateau region was very short because of the strong pore walls of 3D EG sponge
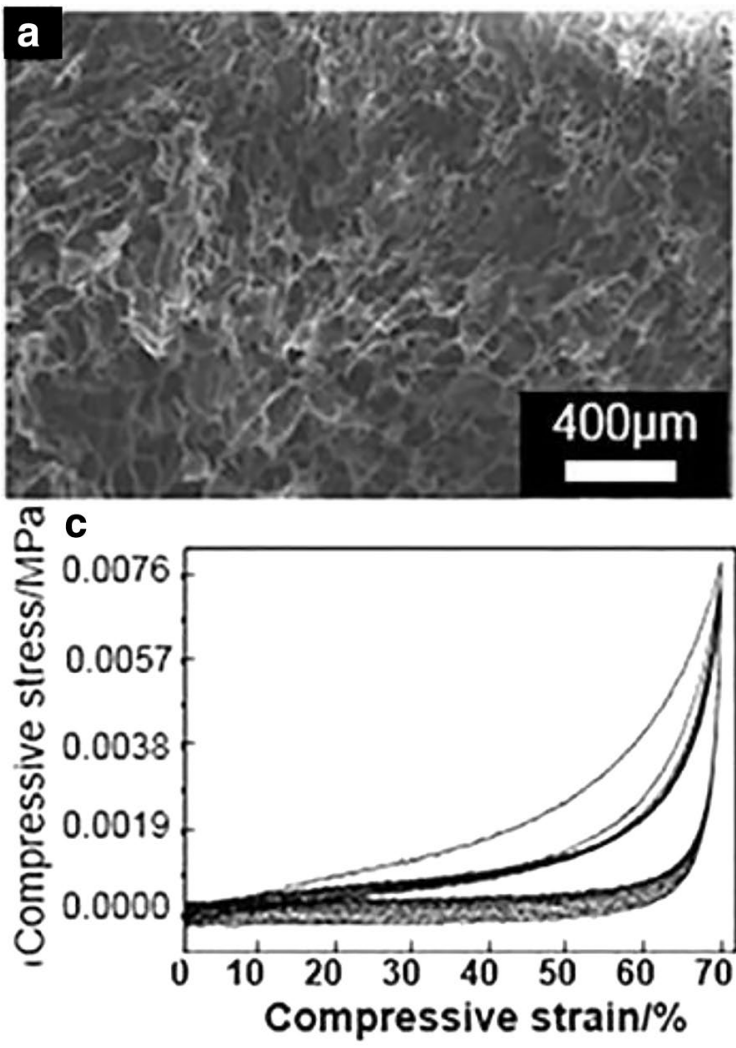

e

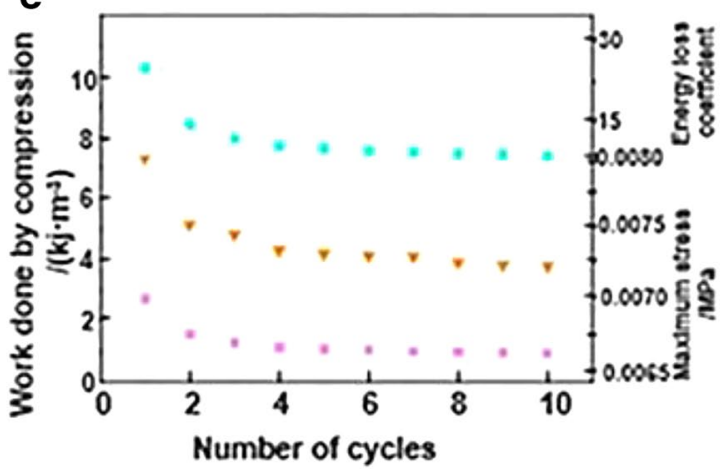

Fig. 6 SEM images of 3D EG sponge with ULGO (a) and LGO (b). Compressive stress-strain curves of 10 cycles of loading and unloading of 3D EG sponge with ULGO (c) and LGO (d). Work done by compression (pink squares), maximum stress at $70 \%$ strain
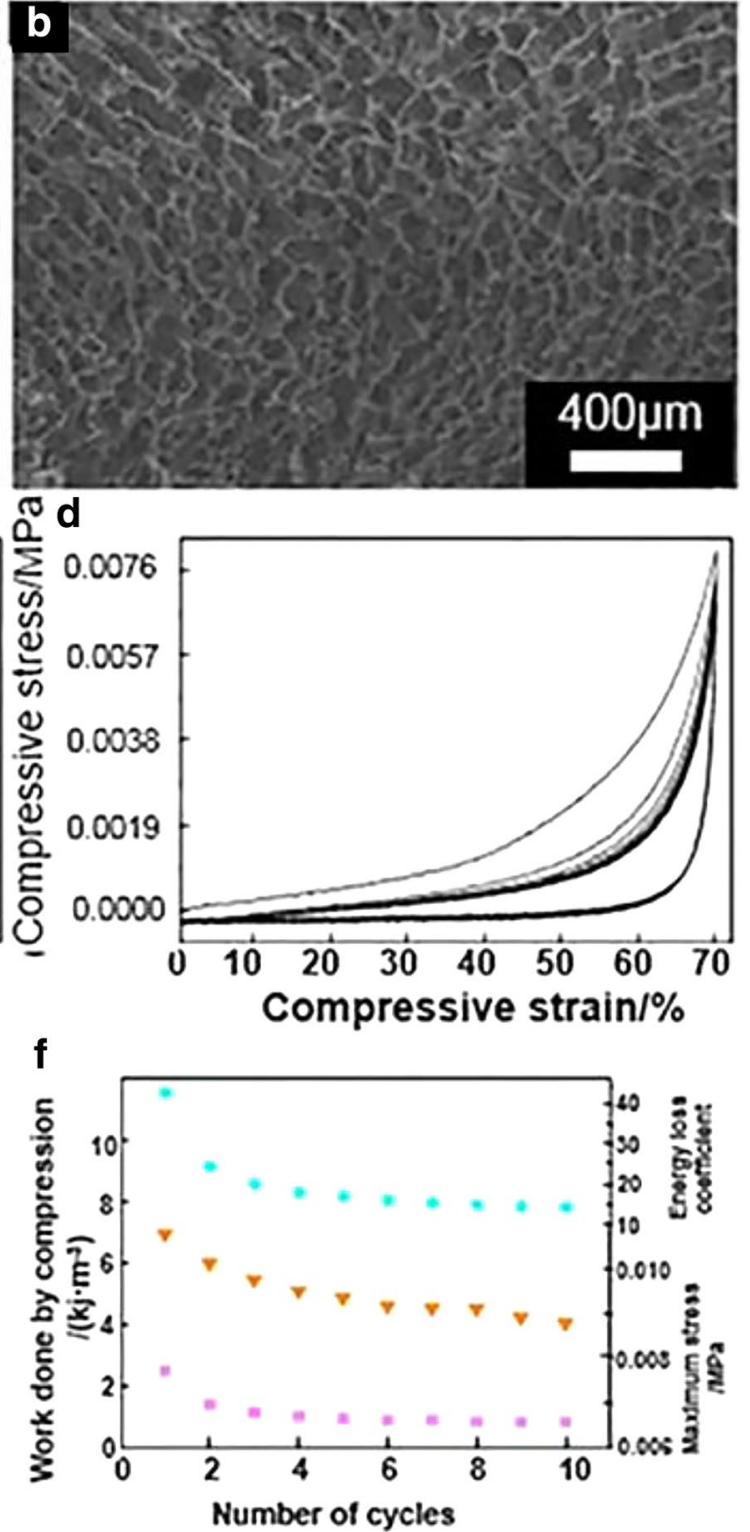

(orange triangles) and energy loss coefficient (blue circles) during the 10 compression cycles in 3D EG sponge with ULGO (e) and LGO (f) 
[28]. The compressive stress was $2.6 \mathrm{kPa}$ at the plateau, and was $8.0 \mathrm{kPa}$ at $70 \%$ strain for the 3D EG sponge prepared by ULGO sheets (Fig. 6c). The compressive stress was $2.6 \mathrm{kPa}$ at the plateau, and was $10.8 \mathrm{kPa}$ at $70 \%$ strain for the 3D EG sponge prepared by LGO sheets (Fig. 6d). In the following compression cycles, the loading curves showed only two regions: a linear region, and a densification region. Thanks to the high strength and the uniform orientation of the pore walls, the contacted walls resisted the attraction of the Van der Waals force with each other, recovering to their original shapes after unloading.

The maximum stress of the 3D EG sponge fabricated by ULGO sheets decreased from 2.5 to $2.2 \mathrm{kPa}$ for 10 cycles. The energy loss coefficient was measured to be $\approx 25 \%$ in the first cycle and 9\% after 10 cycles. In the 3D EG sponge fabricated by LGO sheets, its maximum stress decreased from 3.4 to $2.7 \mathrm{kPa}$. The energy loss coefficient was measured to be $\approx 43 \%$ in the first cycle and $15 \%$ after 10 cycles (Fig. 6e). These results indicated that the 3D EG sponge constructed using ULGO solution had better elastic property. The preparation method extended the application of controlled large GO sheets, but it still had limitation. The technology did not take full advantage of the selfassembly of ULGO sheets. The forming of 3D EG sponge was more complex than fibers and papers. It was difficult to control the reduction process of ULGO sheets into 3D EG sponge with large scale network structure, because of their high flexibility, less functional groups and highly wrinkled topography [29] resulting in unordered stacking of ULGO sheets.

\section{Conclusions}

In summary, in the case of using uniform LGO and ULGO sheets, the nematic LCs phases are formed at low concentration. After chemical reduction, the LCs of GO solution are converted to 3DG sponges with a high degree of orientation, offering a new methodology to regulate the controlled large $\mathrm{GO}$ sheets. The orientation of $\mathrm{GO}$ solution can be inherited by 3DG sponge, making the sponge to have a large-scale ordered network structure. The ordered arrangement of $\mathrm{rGO}$ sheets play an important role in determining the mechanical property of 3DG sponge. The 3D EG sponges have low density and good elasticity, promising for the applications in strain sensing, shock damping, and energy cushioning. This work provides a new method for the preparation of 3DG materials constructed using the LCs of controlled large GO sheets and may shed new light on the relationship between the microstructures and mechanical properties of 3DG sponge.
Acknowledgements This work was financially supported by the key project of Shanghai Science and Technology Committee (No. 14231200300), Shanghai Key Laboratory of Green Chemistry and Chemical Processes and SRF for ROCS, SEM.

\section{Compliance with ethical standards}

Conflict of interest The authors declare that they have no competing interests.

\section{References}

1. Lv W, Zhang C, Li Z, Yang QH (2015) Self-assembled 3D graphene monolith from solution. Chem Lett 6:658-688

2. Jiang L, Fan Z (2014) Design of advanced porous graphene materials: from graphene nanomesh to 3D architectures. Nanoscale 6:1922-1945

3. Yolshina VA, Yolshina LA, Elterman VA, Vovkotrub EG, Shatunova AA, Pryakhina VI, Khlebnikov NA, Tarakina NV (2017) Synthesis of and characterization of freestanding, high-hierarchically structured graphene-nanodiamond films. Mater Des 135:343-352

4. Cong HP, Chen JF, Yu SH (2014) Graphene-based macroscopic assemblies and architectures: an emerging material system. Chem Soc Rev 43:7295-7325

5. Chen Q, Du Y, Li K, Xiao H, Wang W, Zhang W (2017) Graphene enhances the proton selectivity of porous membrane in vanadium flow batteries. Mater Des 113:149-156

6. Dan B, Behabtu N, Martinez A, Evans JS, Kosynkin DV, Tour JM, Pasquali M, Smalyukh II (2011) Liquid crystals of aqueous, giant graphene oxide flakes. Soft Matter 7:11154-11159

7. Xu Z, Sun HY, Zhao XL, Gao C (2013) Ultrastrong fibers assembled from giant graphene oxide sheets. Adv Mater 25:188-193

8. Lin XY, Shen X, Zheng QB, Yousefi N, Ye L, Mai YW, Kim JK (2012) Fabrication of highly-aligned, conductive, and strong graphene papers using ultralarge graphene oxide sheets. ACS Nano 6:10708-10719

9. Wang X, Liu X, Yuan H, Liu H, Liu C, Li T, Yan C, Yan X, Shen C, Guo $Z$ (2018) Non-covalently functionalized graphene strengthened poly(vinyl alcohol). Mater Des 139:372-379

10. Chu K, Wang X, Li Y, Huang D, Geng Z, Zhao X, Liu H, Zhang H (2018) Thermal properties of graphene/metal composites with aligned graphene. Mater Des 140:85-94

11. Wang MS, Huang YL, Wang YY, Dai LY (2016) The effect of tunable graphene oxide sheet size on the structures and catalytic properties of three dimensional reduced graphene oxide sponge. RSC Adv 6:112086-112091

12. Gao YD, Kong QQ, Liu Z, Li XM, Chen CM, Cai R (2016) Graphene oxide aerogels constructed using large or small graphene oxide with different electrical, mechanical and adsorbent properties. RSC Adv 6:9851-9856

13. Yao B, Chen J, Huang L, Zhou Q, Shi G (2016) Base-induced liquid crystals of graphene oxide for preparing elastic graphene foams with long-range ordered microstructures. Adv Mater 28:1623-1629

14. Xu Z, Gao C (2011) Aqueous liquid crystals of graphene oxide. ACS Nano 5:2908-2915

15. Aboutalebi SH, Gudarzi MM, Zheng QB, Kim JK (2011) Spontaneous formation of liquid crystals in ultralarge graphene oxide dispersions. Adv Funct Mater 21:2978-2988

16. Akbari A, Sheath $P$, Martin ST, Shinde DB, Shaibani M, Banerjee PC, Tkacz R, Bhattacharyya D, Majumder M (2016) Largearea graphene-based nanofiltration membranes by shear 
alignment of discotic nematic liquid crystals of graphene oxide. Nat Commun 7:1-9

17. Zhang S, Li Y, Sun J, Wang J, Qin C, Dai L (2016) Size fractionation of graphene oxide sheets assisted by circular flow and their graphene aerogels with size-dependent adsorption. RSC Adv 6:74053-74060

18. Bates MA, Frenkel D (1999) Nematic-isotropic transition in polydisperse systems of infinitely thin hard platelets. J Chem Phys 110:6553-6559

19. Eppenga R, Frenkel D (1984) Monte Carlo study of the isotropic and nematic phases of infinitely thin hard platelets. Mol Phys 52:1303-1334

20. Xu Z, Gao C (2011) Graphene chiral liquid crystals and macroscopic assembled fibres. Nat Commun 2:1145-1154

21. Kim JE, Han TH, Lee SH, Kim JY, Ahn CW, Yun JM, Kim SO (2011) Graphene oxide liquid crystals. Angew Chem Int Ed 50:3043-3047

22. Su CY, Xu Y, Zhang W, Zhao J, Tang X, Tsai CH, Li LJ (2009) Electri$\mathrm{cal}$ and spectroscopic characterizations of ultra-large reduced graphene oxide monolayers. Chem Mater 21:5674-5680

23. Narayan R, Kim JE, Kim JY, Lee KE, Kim SO (2016) Graphene oxide liquid crystals: discovery, evolution and applications. Adv Mater 28:3045-3068
24. Kooij FMVD, Beek DVD, Lekkerkerker HNW (2001) Isotropicnematic phase separation in suspensions of polydisperse colloidal platelets. J Phys Chem B 105:1696-1700

25. Chen W, Yan L (2011) In situ self-assembly of mild chemical reduction graphene for three-dimensional architectures. Nanoscale 3:3132-3137

26. Qiu L, Liu JZ, Chang SLY, Wu Y, Li D (2012) Biomimetic superelastic graphene-based cellular monoliths. Nat Commun 3:187-190

27. Liu T, Huang M, Li X, Wang C, Gui C, Yu ZZ (2016) Highly compressible anisotropic graphene aerogels fabricated by directional freezing for efficient absorption of organic liquids. Carbon 100:456-464

28. Li YR, Chen J, Huang L, Li C, Hong JD, Shi G (2014) Highly compressible macroporous graphene monoliths via an improved hydrothermal process. Adv Mater 26:4789-4793

29. Jalili R, Aboutalebi SH, Esrafilzadeh D, Konstantinov K, Moulton SE, Razal JM, Wallace GG (2013) Organic solvent-based graphene oxide liquid crystals: a facile route toward the next generation of self-assembled layer-by-layer multifunctional 3D architectures. ACS Nano 7:3981-3990 